\title{
3D Printing of Personalized Archwire Groove Model for Orthodontics: Design and Implementation
}

\author{
Gang Liu ${ }^{1}$ \\ State Key Laboratory of Solid Lubrication, Institute of \\ Chemical Physics \\ Chinese Academy of Sciences, School of Information \\ Science and Engineering Lanzhou University \\ Lanzhou,China \\ He Qin ${ }^{2}$ \\ School of Information Science and Engineering \\ Lanzhou University, Lanzhou, China
}

\author{
Haiyan Zhen ${ }^{3}$ \\ First Hospital of Lanzhou University \\ Lanzhou, China
}

\author{
Bin $\mathrm{Liu}^{4}$ \\ School/Hospital of Stomatology \\ Lanzhou University \\ Lanzhou, China
}

\author{
Xiaolong Wang ${ }^{* 5}$ \\ State Key Laboratory of Solid Lubrication \\ Institute of Chemical Physics, Chinese Academy of \\ Sciences \\ Lanzhou, China
}

\author{
Xinyao $\mathrm{Tao}^{6}$ \\ Neoglory Holdings Group \\ Yiwu, China
}

\begin{abstract}
In traditional dental treatment, archwires are bent by orthodontists using standard methods. However, the standard models cater to patients with common oral problems, and are unsuitable for personalized orthodontic treatment, which is highly desired in many cases. A method to prepare a personalized archwire groove model is, undoubtedly, useful for orthodontic treatment in clinical diagnosis. In this study, a threedimensional (3D) printing technology is demonstrated to achieve the personalized archwire groove model in a rapid, computed tomography image compatible manner, to assist orthodontists. This method is expected to improve the efficiency and accuracy of archwire bending and the resultant product can distribute the uniform dentofacial stress, improve the wearing comfort of the patient and further shorten the period of treatment and repair of the tooth.
\end{abstract}

Keywords-3D Printing; personalized; archwire groove model; orthodontic treatment

\section{INTRODUCTION}

With the development of 3D printing technology, the clinical orthodontic effect can be better realized by constructing different individualized models. In orthodontic treatment, archwires are the vital and motivating parts of an orthodontic appliance. Wires are bent and attached to teeth to align them via elastic recovery [1], and they store and deliver power through the brackets and bands to the teeth and surrounding tissues. A good archwire forming technique is an essential part of quality orthodontic treatment [2]. Clinicians normally adopt standard procedures and bending methods causing problems such as poor correction effect and increasing consultation hours because of the mismatch between individual needs and standardized clinical techniques. Therefore, in terms of diagnosis [3], treatment planning and mechanical therapy, strictly following standardized procedures is not suitable nowadays for personalized precise treatment, which therefore, needs to be improved.

According to historical records [4,5], standard dental archwire has been studied for many years and several types have been formed which are commonly used in clinics, such as Vari-Simplex, Tru-arch, Bonwill-Hawley and Brader. The vital elements of an archwire are both its size and shape. It is simple and easy to classify the standard archwire into large, medium and small on the basis of its size [6], but this classification ignores the shape factor. Some drawbacks are found, on analyzing several cases, in the types of archwire mentioned: the Vari-Simplex arch, designed by Alexander, is not adaptive to special patients because of its inaccurate classification; the Truarch, designed by Roth, has an obvious danger of anterior dental arch expansion and posterior dental arch shrink in clinical applications, because it widens the front arch radian (especially in the premolar regions) and shrinks the posterior arch radian, which leads to a $3 \mathrm{~cm}$ wide difference on each side; the Bonwill-Hawley arch, designed by Hawley, has two characteristics viz. one is a proper front radian for incisor and canine teeth and the other is by using line segments to represent premolars and molars. But, it also has very apparent disadvantages such as low coincidence rate with normal dental arch, as gleaned from a large amount of literature, lacks aesthetic appearance. Using the mathematical model of a triangular ellipse, Brader designed the Brader arch which is effective in narrowing the canine area during clinical application, according to available literature, because Brader only considered the size. It can be seen obviously, that there are still some problems in the existing standard archwires.

Meanwhile, it could be found, by considering orthodontic clinical cases over the years, that applying the same kind of archwire to all patients did not get satisfactory results, hence a

*Corresponding author 
personalized approach should be adopted [7]. Some dentists found that it was important to choose individualized archwire according to the dental shapes of the patient, after straight-wire appliances had been used in the clinic for 20 years [8], failing which, it was easy to cause recidivism and loosening of the tooth. The size of the personalized archwire is not the only important concern; in addition, the chair-side time would be largely reduced during each return visit [9] and the time span between two return visits would be extended. Moreover, the holistic shift of the teeth is more effective and the occluding relation is more normal so that the alignment period would be reduced. In this way, the clinicians could quickly control overbite, reduce overjet and close the space[10,11], which would shorten the therapy time and spare dentists more time to make intense adjustment of the teeth and enhance the curative effect.

In recent years, with the rapid development of 3D printing, it is possible to convert a digital model into a solid one in a short time [12-14]. Using this kind of information engineering technology, clinicians can perform the study of real-time virtual correction effects on patients with malocclusion deformity and carry out orthodontic clinical practice [15]. Aided by 3D printing technology [16-20], clinicians can design and truly achieve the personalized archwire model with minimum difference.

The purpose of this study is to construct and print a personalized archwire groove model, using 3D printing technology, which can assist orthodontists in shaping personalized archwire rapidly thus improving the efficiency and accuracy of archwire bending. In addition, this method can distribute the uniform dentofacial stress and improve the wearing comfort of the patient and further shorten the period of treatment and repair of the tooth.

\section{MATERIALS AND METHODS}

\section{A. Materials}

A male patient, who had voluntarily joined the study and given prior consent was selected as the experimental subject. A CBCT (cone-beam computerized tomography) scanner (KaVo 3D eXam 5, KaVo Corporation, Germany, layer thickness 0.25 $\mathrm{mm}$, exposure time $14.7 \mathrm{~s}$ ) was used to obtain several DICOM (Digital Imaging and Communications in Medicine) formats of the lower jaw teeth data of the patient. The "Import Images" tool of Mimics software was then used to import these DICOM format files and from the original images, graphs of coronal plane and sagittal plane were automatically calculated and generated through Mimics. The location of these pictures is shown by Mimics using three views, which are related to each other and can be rapidly located through the mouse and location toolbar. The corresponding tissue pixels were extracted through the threshold, and put in a mask (Mask) and the Draw and Erase tools were utilized to edit and modify the mask, so as to extract the required image of the teeth and jaw bone tissue. Subsequently, an STL (Stereography) file format of 3D model was generated by using Export Binary STL command, i.e. 2D scan images were converted into 3D entities successfully.
The constructed STL format 3D mandible model was loaded into Geomagic where the repair of the model was carried out. The mandible model after filling the hole approximately presented a rough model, on which the fairing operation had to be performed by fast fairing command. After completion of the editing processing of the polygon, the shape had to be refined with operations like detection of curvature, construction and editing patches, construction of the grid and fitting surface, which was used for reconstruction of NonUniform Rational B-Splines (NURBS) surface of the mandible. The building patches of the mandible were completed by performing the "upgrade / constraint" command based on the Andrews six keys theory of maxillofacial coordination [21, 22], landmarks were selected on the anlagen bone surface, and personalized archwire was then drawn to guide the orthodontic clinical work. The steps for the selection of a range of personalized archwire grooves are as follows:

1) Selection of the corresponding landmarks: In this study, the landmarks were selected mainly from one-third dental root, i.e., reference points of forming the archwire from the outside and inner part of the tooth jaw, in order to provide a reasonable selection range for orthodontists who traditionally selected landmarks on the crown surface of the molar and canine. The reference point from one-third dental root of the sixth teeth was selected and the horizontal plane parallel to it was constructed before adjusting the grid size of the plane from the angle of view for selecting the key points. The selected point data are as shown in Table 1.

TABLE I. CoORdinate VAlues of Point Data

\begin{tabular}{|c|c|c|c|}
\hline \multicolumn{2}{|c|}{$\begin{array}{l}\text { Coordinate values of point } \\
\text { data } \\
\text { on external maxillofacial }(\mathrm{mm})\end{array}$} & \multicolumn{2}{|c|}{$\begin{array}{l}\text { Coordinate values of point data } \\
\text { on internal maxillofacial }(\mathbf{m m})\end{array}$} \\
\hline $\mathbf{x}$ & $\mathbf{y}$ & $\mathbf{x}$ & $\mathbf{y}$ \\
\hline 5.4850 & 46.3128 & 16.0828 & 48.7771 \\
\hline 9.4565 & 34.7752 & 19.6157 & 39.0663 \\
\hline 12.5626 & 26.3565 & 22.0526 & 30.6267 \\
\hline 15.8220 & 19.3156 & 23.4347 & 23.3133 \\
\hline 20.4598 & 13.1289 & 25.9774 & 17.6465 \\
\hline 26.3904 & 8.7769 & 28.7833 & 13.7254 \\
\hline 33.3653 & 7.0508 & 33.3608 & 11.4007 \\
\hline 38.4456 & 6.6948 & 38.7544 & 12.1141 \\
\hline 45.5535 & 8.0638 & 43.5308 & 13.1859 \\
\hline 51.3331 & 11.3389 & 47.5262 & 17.3475 \\
\hline 57.0771 & 16.7109 & 50.3400 & 22.4378 \\
\hline 60.7823 & 23.6831 & 52.4883 & 30.0677 \\
\hline 63.9181 & 31.4431 & 54.9923 & 38.0855 \\
\hline 67.7258 & 43.3829 & 57.5778 & 46.4019 \\
\hline
\end{tabular}

2) Determination of the range of personalized archwire groove

Research findings from relevant literature [23] have been referred to. The fitting curve of dental arch was drawn by the following mandibular Equation (1).

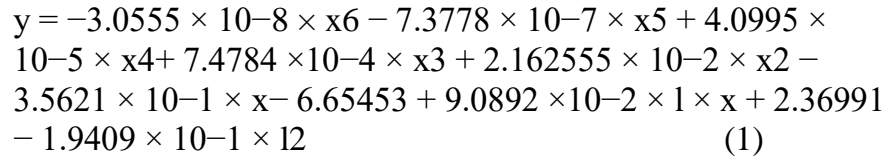




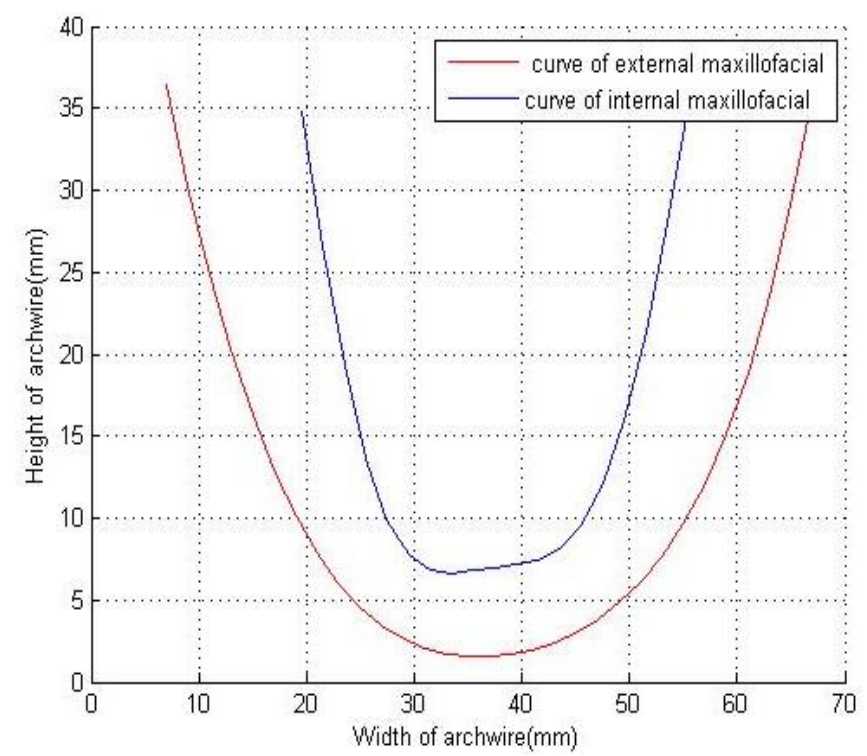

Fig 1. Selected Range Of Personalized Archwire Groove.

Where, 1 is the average width value of left and right central incisor crown.

The mesiodistal maximum diameter of mandibular central incisor crown from the patient's model was obtained by using dividers. If the values of the width of the left and right central incisor crown were obviously inconsistent, the mean value was taken. When the coordinate values of point data, as shown in Table 1, were imported into the computer, the output of the dental arch i.e. the range of personalized archwire groove was acquired immediately, as shown in Fig. 1.

\section{B. Methods}

After the designed 3D solid model of the teeth of the patient was loaded into ANSYS, as shown in Fig. 2(a), the working plane with grid size was constructed, and key points were selected around the teeth in the range of the working plane shown in Fig. 2(b), after which they were connected by using the Spline through KPs command under Extrude tools, and the bow- shaped curve was obtained, as shown in Fig. 2(c).

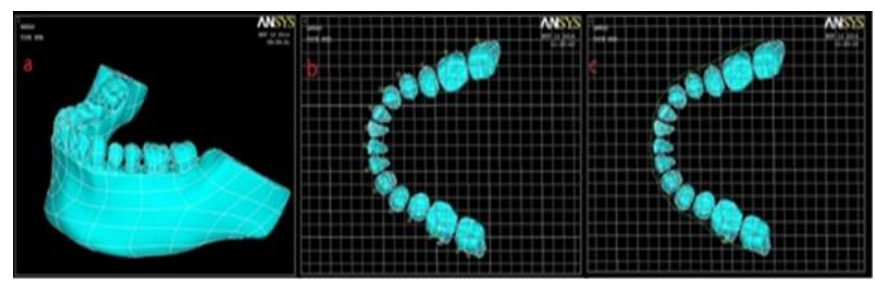

Fig 2. Construction of Personalized Archwire Groove (a) 3D Solid Model; (b) Key Points; (c) Formation of Archwire Line.

In the design of the archwire slot model to meet personalized needs, a key factor to be considered is the physical properties of wire materials. Orthodontic wires made of metal usually have good elasticity and rebound while being bent. The concrete shape of the archwire groove needs to be calculated according to the bow parameters, which would enable the archwire to gain a preset accurate shape after springback.
TABLE II. SPRINGBACK CURVE OF Nickel TITANIUM Alloy ARChWiRE

\begin{tabular}{|c|c|}
\hline Bending angle $\left(^{(}\right)$ & Spingback angle $\left(^{{ }^{\circ}}\right)$ \\
\hline 0 & 0 \\
\hline 20 & 6 \\
\hline 40 & 7.4 \\
\hline 60 & 9 \\
\hline 80 & 10.5 \\
\hline 100 & 12.7 \\
\hline 120 & 15 \\
\hline
\end{tabular}

Nickel titanium (NiTi) alloy was used to implement the subsequent archwire bending experiment, because this material has excellent mechanical properties including super elasticity, shape memory effect and damping characteristics. Its elastic limit is far greater than ordinary materials and it does not obey Hooke's law during increasing stress range of deformation with the increase of strain, therefore, it is commonly used in making orthodontic wires.

In order to measure the springback angle of archwires made of this material at different bending angles, an experiment was specially designed. The archwire was arranged and fixed on a base plate and vertical pressure was applied on it at a distance of $2 \mathrm{~mm}$ on the right side of the base plate where $\theta$, the bending angle, is the angle between the tangent line of bending arc and horizontal line before bending and $\alpha$, the rebound angle, is the angle between the tangent line of bending arc before and after the archwire rebound.

The experimental results (as shown in Table 2) indicate that the springback angle increases with the increase of bending angle when the bending angle is greater than 10 degrees, which is close to the law of linear growth.

Combined with the data of dental arch of patients already obtained, some discrete key points at intervals of $2 \mathrm{~mm}$ were selected from the arch curve and the angle composed of three adjacent discrete points, which was the plastic deformation angle, was calculated. The bending angle based on the rebound curve of archwire and the plastic deformation angle which is the difference between bending angle and the springback angle, were also worked out. Through coordinate transformation of all angles, the key points of the personalized archwire groove were finally determined and the archwire thread based on these key points was drawn.

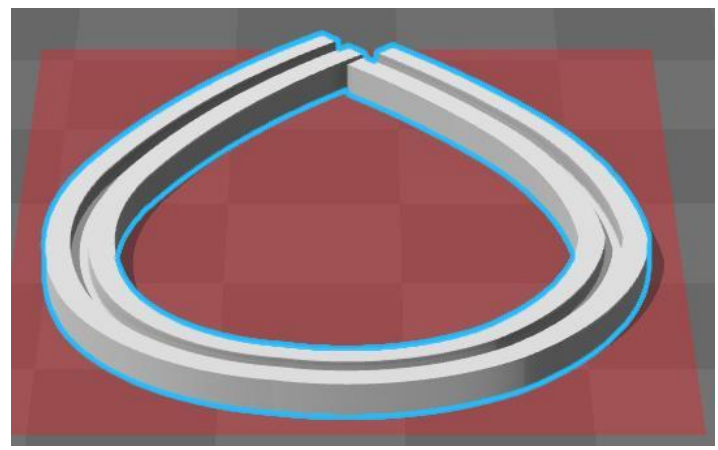

Fig 3. 3D Solid Model of Personalized Archwire Groove. 
In this way, a 3D solid model of an archwire groove was shaped (as shown in Fig. 3) after construction of the working plane based on key points at the end and drawing the archwire groove on it.

\section{RESULTS AND DiSCUSSION}

The personalized archwire groove model was printed out by the 3D printer (MakerBot Company, American) and the archwire was bent by using this groove model (as shown in Fig. 4).

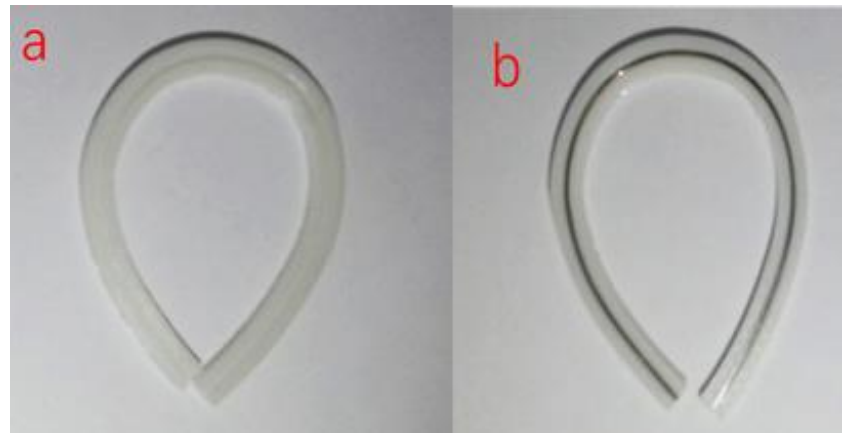

Fig 4. (a) 3D Printed Archwire Groove Model and (b) Bent Archwire.

Meanwhile, a wire bent by the archwire groove model was compared with that bent by the standard drawing method, by using a wire of 0.016 " diameter. Fig. 5(a) represents the archwire of the test group using the method illustrated in this study, and Fig. 5(b) represents that of the control group using the standard method. It is obvious that the opening degree of the wire in Fig. 5(a) is smaller than that in Fig. 5(b), and is closer to the actual oral situation in patients.

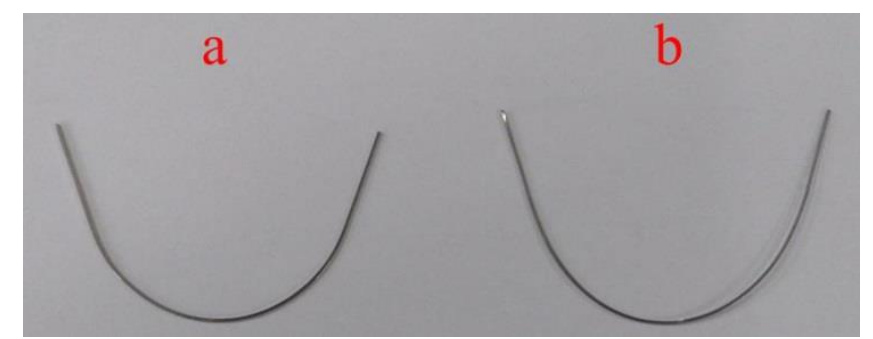

Fig 5. Archwire Bent with (a) 3D Printed Model and (b) Standard Method.

In order to further verify the advantages of personalized archwire groove model, the author specially displayed the individual dental arch model of the patient and the standardized dental arch model in the same coordinate system and carried out the test of goodness of fit by means of Equation (2). The goodness of fit values are shown in Table 3.

$$
\mathrm{R}^{2}=1-\frac{\sum_{i=1}^{n}\left(y_{i}-\hat{y}_{i}\right)^{2}}{\sum_{i=1}^{n}\left(y_{i}-\bar{y}\right)^{2}}
$$

Where, yi is the value of dental arch in the patient; $y$ is the average value of dental arch in the patient; $\hat{y} i$ is value of the different kinds of archwires.

It can be seen from Table 3 that the dental arch form in the test group was similar to that of patients in the control group.
TABLE III. GOODNESS OF FIT VALUES

\begin{tabular}{|c|c|}
\hline Group & $\begin{array}{c}\text { Goodness of fit values between the actual } \\
\text { archwire and group }\end{array}$ \\
\hline a group & 0.999446 \\
b group & 0.994317 \\
\hline
\end{tabular}

To summarize, the results showed that the idea of designing a personalized archwire groove was viable. In theory, the performance and feasibility of the archwire groove as an application of $3 \mathrm{D}$ printing technology in orthodontics was tested.

The method based on Mimics and ANSYS software was developed specifically for designing the archwires groove. There were several drawbacks in the software that need to be addressed. Firstly, the complete archwires groove design process depended on commercial software (Geomagic and Mimics) and required human interaction, which reduced its efficiency. In future, special software should be developed to enable an automated design process. Secondly, the threshold for preprocessing DICOM format teeth data required to be manually set and adjusted; in addition, although the collection of key points to form the archwire could be selected manually in the visual condition, the accuracy needs to be improved.

However, the key point of orthodontic digitization research was its application to clinical orthodontics after obtaining the complete digital information. In previous research, the arrangement of the tooth crown was a more developed three-dimensional way of arrangement of teeth, and almost every commercial orthodontic virtual treatment software and the arrangement adopted in the present study were based on this, but the ideal position of the root of the tooth was not considered in this method, in other words, the key factor that influenced the stability and functionality was ignored. The concept of the treatment was limited to the tooth crown, but the relationships between the root of the tooth and supporting bone, the tooth and lip, as well as other soft tissues were ignored. The application of the integrated digital model data which includes the information obtained from cone beam computed tomograms (CBCT) of the tooth root and jawbones can deal effectively with many problems brought on by the previous virtual arrangement of teeth, avoiding many problems such as periodontal fenestration defects and fracture, which result in the process of aligning the dentition to be more applicative to the anatomical physiology characteristics of humans.

\section{CONCLUSIONS}

In conclusion, the methods of personalized archwire groove model design were analyzed and explained, which examined its substantial role in further promoting 3D printing technology in the application of orthodontic treatment. However, the design result needs to be precisely modified using the tools available in the two software packages to obtain a satisfactory shape. In addition, the entire 3D printing process was completed through human-computer interaction. Therefore, further research is necessary in automated design software to make archwire groove model design both simpler and faster. 


\section{ACKNOWLEDGMENTS}

This work was supported by the Natural Science Foundation of Gansu Province, China (145RJZA126, 1606RJZA051 and 17JR5RA318), the Fundamental Research Funds for the Central Universities (grant number lzujbky-2015102), and the Western Project of CAS.

\section{REFERENCES}

[1] R.J. Nikolai, Orthodontic wire: a continuing evolution. Semin. Orthod. Vol. 3. pp. 157-165,1997.

[2] J. Ferčec, I. Anžel, R. Rudolf, Stress dependent electrical resistivity of orthodontic wire from the shape memory alloy NiTi, Mater. Des. Vol.55,pp.699-706,2014 .

[3] S.M. Castro, M.J. Ponces, J.D. Lopes, M. Vascomcelos, M.C.F. Pollmann, Orthodontic wires and its corrosion-The specific case of stainless steel and beta-titanium, J. Dent. Sci. Vol.10, pp.1-7, 2015.

[4] E.A. Begole, Computer based methodology for construction of orthodontic arch wire templates, Comput. Prog. Bio. Vol.19, pp.61-68, 1984.

[5] X. Li, J. Wang, E.H. Han, W. Ke, Influence of fluoride and chloride on corrosion behavior of NiTi orthodontic wires, Acta Biomater. Vol. 3, pp. 807-815, 2007.

[6] A.A. Nasef, A.R. El-Beialy, Y.A. Mostafa, Virtual techniques for designing and fabricating a retainer, Am. J. Orthod. Dentofacial. Orthop. Vol.146, pp.394-398, 2014.

[7] F. Yuan, Y. Sun, Y. Wang, P. Lv, Computer-aided design of tooth preparations for automated development of fixed prosthodontics, Comput. Biol. Med. Vol.44, pp.10-14,2014 .

[8] G. N. Boone, Archwires designed for individual patients, Angle Orthod. Vol.33,pp.178-185, 1963 .

[9] M. Salmi, K.S. Paloheimo, J. Tuomi, J. Wol, A. Makitie, Accuracy of medical models made by additive manufacturing (rapid manufacturing) J. Cranio. Maxill. Surg. Vol.41,pp.603-609, 2013 .

[10] S. C. Ligon, R. Liska, J. Stampfl, M. Gurr, and R, Mülhaupt, cy of medical models made by additive manufacturing (rapid manufacturing) J. Cranio. Maxill. Surg. he Cent
[11] T.M. Rankin, N.A. Giovinco, D.J. Cucher, G. Watts, B. Hurwitz, D.G. Armstrong, Three-dimensional printing surgical instruments: are we there yet? J. Surg. Res. Vol.189, pp.193-197, 2014 .

[12] B. Berman, 3-D printing: The new industrial revolution, Bus. Horiz. Vol.55, pp. 155-162, 2012

[13] Y. Zhang, S. Zuo, J. Jiang, Y. Liu, Y. Liu, Interactive adjustment of individual orthodontic archwire curve, Chin. J. Sci. Instrum. Vol.38, pp.1616-1624, 2017.

[14] D.S.C. Soon, M.P. Chae, C.H.C. Pilgrim, W.M. Rozen, R.T. Spychal, D.J. Hunter-Smith. 3D haptic modelling for preoperative planning of hepatic resection: A systematic review, Ann. Med. Surg. Vol.10, pp. 1-7, 2016 .

[15] Birtchnell T, Urry J. 3D, SF and the future, Futures, 50 (2013) 25-34.C. Growth, N. D. Kravitz, and J. M. Shirck, "Incorporating threedimensional printing in orthodontics", J Clin Orthod. 2018, pp. 28-33, 2018.

[16] A. Sutradhar, J. Park, D. Carrau, M.J. Miller, Experimental validation of 3D printed patient-specific implants using digital image correlation and finite element analysis, Comput, Biol. Med. Vol.52, pp. 8-17, 2014 .

[17] H.T. Yau, T.J. Yang, Y.C. Chen, Tooth model reconstruction based upon data fusion for orthodontic treatment simulation, Comput. Biol. Med. Vol.48, pp.8-16, 2014 .

[18] M. Gebler, A.J.M.S. Uiterkamp, C. Visser, A global sustainability perspective on 3D printing technologies, Energy Policy . Vol.74, pp.158-167, 2014.

[19] S. Bose, S. Vahabzadeh, A. Bandyopadhyay, Bone tissue engineering using 3D printing, Mater. Today. Vol.16, pp. 496-504, 2013.

[20] G. Chen, W. Fan, S. Mishra, M.J. Miller, Tooth fracture risk analysis based on a new finite element dental structure models using micro-CT data, Comput. Biol. Med. Vol.42, pp. 957-963, 2012.

[21] P. Shah, and B. S. Chong, , M.J. Miller, Tooth fracture risk anal planning in Endodontics", Clinical oral investigations. pp. 1-14, 2018.

[22] D. Rejeski, F. Zhao, Y. Huang, . Miller, Tooth fracture risk anal planning in Endodontics", Clinical oral investigations. pp. 1-14, 2018.T data, Comput. Biol. Med. a

[23] J. Qiu, L. Han, J. Li, H. Li, The study of mathematics model of customized dental arch in Han race of young adult in Tianjin, Chin. J. Prosthodont. Vol.15, pp. 6-10, 2014. 